\title{
Implementing the three-equation method of measuring single breath carbon monoxide diffusing capacity
}

\author{
BRIAN L GRAHAM PhD, JOSEPH T MINK BSc, DAVID J COTTON MD FRCPC \\ Division of Respiratory Medicine, Department of Medicine, University of Saskatchewan, \\ Saskatoon, Saskatchewan
}

BL GRAHAM, JT MINK, DJ COTTON. Implementing the three-equation method of measuring single breath carbon monoxide diffusing capacity. Can Respir J 1996; 3(4):247-257.

Conventional methods of measuring the single breath diffusing capacity of the lung for carbon monoxide (DLco ${ }^{\mathrm{SB}}$ ) are based on the Krogh equation, which is valid only during breath holding. Rigid standardization is used to approximate a pure breath hold manoeuvre, but variations in performing the manoeuvre cause errors in the measurement of $\operatorname{DLco}^{\mathrm{SB}}$. The authors previously described a method of measuring DLco ${ }^{\mathrm{SB}}$ using separate equations describing carbon monoxide uptake during each phase of the manoeuvre: inhalation, breath holding and exhalation. The method is manoeuvre-independent, uses all of the exhaled alveolar gas to improve estimates of mean DLco ${ }^{\mathrm{SB}}$ and lung volume, and is more accurate and precise than conventional methods. A slow, submaximal, more physiological single breath manoeuvre can be used to measure DLco ${ }^{S B}$ in patients who cannot achieve the flow rates and breath hold times necessary for the standardized manoeuvre. The method was initially implemented using prototype equipment but commercial systems are now available that are capable of implementing this method. The authors describe how to implement the method and discuss considerations to be made in its use.

Key Words: Diffusion, Diffusing capacity, Gas exchange, Lung volume, Transfer factor
Utilisation de la méthode à trois équations pour mesurer la capacité de diffusion du monoxyde de carbone en apnée

RÉSUMÉ : Les méthodes traditionnelles pour mesurer la capacité de diffusion du monoxyde de carbone (DLco) en apnée sont basées sur la formule de Krogh, qui est valide uniquement pendant l'apnée. On procède alors à une normalisation arbitraire pour se rapprocher de la manoeuvre en apnée pure, mais les variations qui se produisent en pratiquant la manoeuvre sont une source d'erreur dans la mesure de la DLco en apnée. Les auteurs ont précédemment décrit une méthode de mesure de la DLco en apnée où l'on utilisait des équations séparées décrivant la capture du monoxyde de carbone pendant chaque phase de la manoeuvre : inspiration, apnée et expiration. La méthode est indépendante de la manoeuvre, utilise la totalité du gaz alvéolaire expiré pour améliorer les estimations de la DLco moyenne en apnée et du volume pulmonaire, et est plus fiable et précise que les méthodes traditionnelles. Une manoeuvre en apnée lente, sous-maximale et plus physiologique peut être utilisée pour mesurer la DLco en apnée chez les patients qui ne peuvent atteindre les débits et rester en apnée pendant le temps nécessaire à la manoeuvre normalisée. La méthode a été initialement développée en utilisant un matériel prototype, cependant des appareils commerciaux sont maintenant disponibles pour permettre la mise en application de cette méthode. Les auteurs décrivent comment procéder et discutent des considérations relatives à son utilisation. 
$\mathrm{T}$ he single breath diffusing capacity of the lung for carbon monoxide (DLco ${ }^{\mathrm{SB}}$ ) was first measured by Krogh (1), who developed an equation to describe the uptake of carbon monoxide during breath holding. Conventional methods of measuring DLco ${ }^{\mathrm{SB}}$ still use the Krogh equation (1) and are therefore constrained by the requirement of a rigidly standardized single breath manoeuvre, which approximates a pure breath hold but obviously includes inhalation and exhalation. Although the uptake of carbon monoxide by the lungs measured in this manner is more appropriately called the transfer factor (2), we have used the American Thoracic Society (ATS) convention of calling it diffusing capacity (3).

Most current clinical measurements of DLco ${ }^{\mathrm{SB}}$ use some variation of the technique described by Ogilvie et al (4). The Jones and Meade method (5) applies corrections to the timing of the breath hold period and recommends the collection of a very small alveolar gas sample immediately following dead space washout. The method recommended by Ferris (6), referred to as Epidemiology Standardization Project method, appears to have been developed for ease of computation and standardization without a theoretical or clinical rationale. We have previously shown that all of these methods overestimate DLco $^{\text {SB }}$ measured from a lung model (7) and normal subjects (8), particularly when the exhaled flow rates are reduced.

DLco $^{\mathrm{SB}}$ is overestimated by conventional methods because they are based on a single equation that is valid only during breath holding. The methods rely on the use of a standardized manoeuvre consisting of rapid inhalation, $10 \mathrm{~s}$ of breath holding and rapid exhalation to approximate a pure breath hold manoeuvre $(2,3)$. However, the measurement of DLco $^{\mathrm{SB}}$ depends on how well the subject can perform the manoeuvre. If the inspiratory or expiratory flow rates are reduced, if the breath hold time is changed, if the alveolar sample is collected later in exhalation, or if the volume of the alveolar sample is changed, then the measured DLco ${ }^{\mathrm{SB}}$ will be spuriously affected (7-9).

For normal subjects who can inhale and exhale with high flow rates and hold their breath for $10 \mathrm{~s}$, measurement of DLco $^{\mathrm{SB}}$ is not a problem because the differences between the various methods are small (9). For patients with airflow obstruction, or any other subject who cannot adequately perform the standardized single breath manoeuvre, DLco ${ }^{\mathrm{SB}}$ is usually overestimated (8).

We developed a method of measuring DLco ${ }^{\mathrm{SB}}$ based on three equations - one each for inhalation, breath holding and exhalation (7). These equations analytically account for the differences in the rate of carbon monoxide uptake during inhalation, breath holding and exhalation. Diffusion of carbon monoxide during all three phases of the manoeuvre inhalation, breath holding and exhalation - can be determined, eliminating the need to assume that all carbon monoxide transfer occurs during breath hold. This makes the measurement of DLco ${ }^{\mathrm{SB}}$ independent of the manoeuvre, and increases precision and accuracy of DLco ${ }^{\mathrm{SB}}$ measurements (9). The subject is not required to inhale and exhale with maximum flow rates and breath hold for $10 \mathrm{~s}$ to approximate a pure breath hold manoeuvre. Theoretically, variations in the flow rates and breath hold times do not cause errors in the measurement of DLco ${ }^{\mathrm{SB}}$.

With the three-equation method, gas concentrations and lung volume are monitored continuously throughout the single breath manoeuvre. These data are used to calculate the carbon monoxide concentrations in the alveolar space and to predict the uptake of carbon monoxide. By comparing the observed with the predicted carbon monoxide uptake, DLco $^{\text {SB }}$ can be calculated. Because the inspiratory flow and gas concentrations are monitored and the carbon monoxide uptake is computed during inhalation, the assumptions for start of breath holding time and carbon monoxide concentration at 'time zero' used by conventional methods are not required.

The three-equation method permits the measurement of DLco $^{\text {SB }}$ at different lung volumes (10), different breath hold times (11) and different flow rates (9). The method also permits all of the exhaled alveolar gas to be used in the calculation of DLco ${ }^{\mathrm{SB}}$. This yields a measurement of DLco ${ }^{\mathrm{SB}}$ that more accurately reflects the mean carbon monoxide uptake of the entire lung. In contrast, conventional methods use smaller alveolar gas samples collected immediately after dead space washout to reduce timing errors, but, unfortunately, the measurements of both the DLco ${ }^{\mathrm{SB}}$ and the absolute lung volume are more influenced by ventilation inhomogeneities in the lung when measured using small gas samples.

We have focused the implementation of this method on the use of a manoeuvre with relaxed flow rates with submaximal lung volumes that can be done equally well by subjects or patients and that more closely approximates normal breathing. The manoeuvre can be done with or without breath holding. The method is by no means restricted to this or any other particular manoeuvre.

The three-equation DLco ${ }^{\mathrm{SB}}$ method was initially developed using a mass spectrometer, a modified infrared absorption type carbon monoxide analyzer and other prototype equipment $(8,9)$. With the continuing refinement of computerized pulmonary function equipment and the improvement of gas analyzers, it is now feasible to use commercial pulmonary function systems to implement this method. We present considerations for the implementation of the method and an efficient computer algorithm for rapid calculation of DLco ${ }^{\mathrm{SB}}$.

\section{EQUIPMENT}

Gas analyzers: While, in theory, the three-equation method could be implemented by using the mean carbon monoxide concentration measured from any size of gas sample collected during exhalation (7), in practice, problems of dead space washout, sample timing and sample volume prevent the advantages of the three-equation method from being fully realized when applied to data collected by conventional DLco $^{\text {SB }}$ equipment (12). The availability of continuously measured concentrations of carbon monoxide and a tracer gas not only facilitates implementation of the three-equation algorithm but also permits simultaneous measurements of ventilation and gas mixing from the same single breath manoeuvre (10). 
TABLE 1

Error in DLco ${ }^{\mathrm{SB}}$ due to carbon monoxide analyzer response time

\begin{tabular}{cccc}
\hline $\mathbf{t}_{\mathbf{0}-90 \%}(\mathbf{m s})$ & $\tau(\mathbf{m s})$ & Shift (points) & Error $(\%)$ \\
\hline 0 & 0 & 0 & 0 \\
67 & 29 & 1 & -0.01 \\
133 & 58 & 2 & -0.22 \\
200 & 87 & 3 & -0.68 \\
267 & 116 & 4 & -0.91 \\
333 & 145 & 5 & -1.13 \\
400 & 174 & 6 & -1.41 \\
467 & 203 & 7 & -1.63 \\
533 & 232 & 8 & -1.87 \\
600 & 261 & 9 & -2.15 \\
667 & 290 & 10 & -2.47 \\
\hline
\end{tabular}

Data from a simulated normal adult lung model using the single breath manoeuvre shown in Figure 5. Response times of carbon monoxide analyzer were simulated to give optimal shifts of 0 to 10 sample points. The error was reduced by an average of $66 \%$ when the breath hold time was increased to $10 \mathrm{~s}$. There was little change in the error when the single breath diffusing capacity of the lung for carbon monoxide (DLCOSB) was changed from 10 through $40 \mathrm{~mL} / \mathrm{min} / \mathrm{torr}$. $t_{0-90 \%}$ is the $0 \%$ to $90 \%$ response time of carbon monoxide analyzer (ms); $\tau$ is the analyzer time constant (ms); shift is the optimal shift in sample points (20 ms); error is the percentage error in the measured $D\left\llcorner C O S B\right.$ compared with the simulated D $\left\llcorner C O^{S B}\right.$

Infrared absorption type analyzers are commonly used for measuring carbon monoxide concentration. Care must be taken to ensure that background carbon dioxide and water vapour do not interfere with the carbon monoxide concentration signal, particularly in view of the very low carbon monoxide levels to be measured. An additional compensation strategy that is incorporated in the algorithm is discussed below.

The response time of the analyzer is also of concern. A slowly responding analyzer will produce inaccurate DLco ${ }^{\mathrm{SB}}$ measurements (Table 1). The response time is generally a function of the size of the sample chamber, the sample aspiration rate, the gas pressure in the sample cell and the geometry of the system. Increasing the aspiration flow rate, which usually decreases the chamber pressure (and hence decreases the density of the test gas in the chamber), will improve the response time, but will decrease the signal to noise ratio. Similarly, using a smaller sample chamber will increase response time but will also diminish the signal to noise ratio. A balance between response time and noise must be found. In the test system described here, a commercially available analyzer was modified to provide a $0 \%$ to $90 \%$ response time of $110 \mathrm{~ms}$ at an aspiration rate of $125 \mathrm{~mL} / \mathrm{s}$ with a root-meansquare noise level of $1 \%$ full scale.

The signal to noise ratio of sampled data increases proportionally with the square root of the number of samples considered. For example, averaging 100 samples to obtain zero level readings improves the signal to noise ratio by a factor of 10 to $0.1 \%$ full scale. There is a similar improvement in determining the mean carbon monoxide concentration in the exhaled gas.

Digital techniques for compensating for the response time

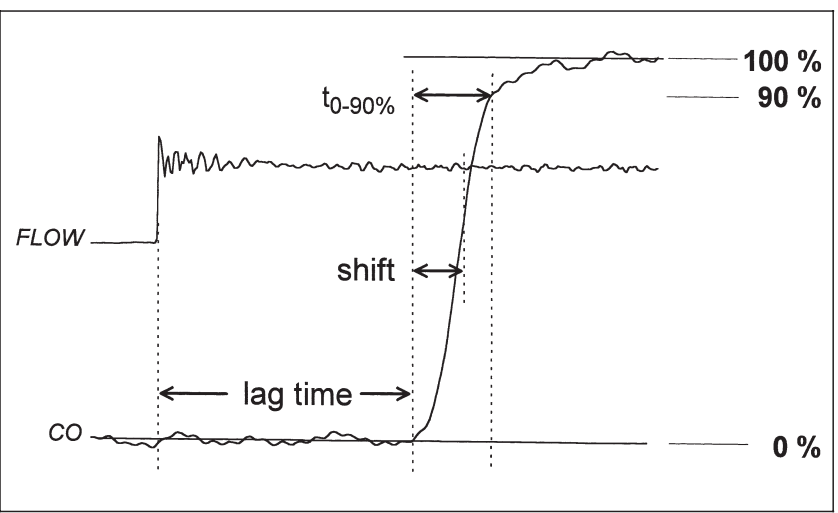

Figure 1) Lag and response times for carbon monoxide: the response time of the analyzer was estimated by rapidly switching the gas being sampled from zero to full scale carbon monoxide. The change in the flow signal shows the time at which the switch was made from medical air to test gas. The lag time, the 0\% to $90 \%$ response time ( $t_{0-90 \%)}$ and the optimal shift are calculated from the resulting response curve. A similar analysis was done for the helium concentration signal

of the gas analyzers are available, but the noise levels typical of infrared analyzers prevent these methods from being very useful. A more suitable compensation technique, given the noise levels and sampling rates used, is an optimal shift of the signal, which is the natural logarithm of twice the time constant (13).

The lag time of the analyzer, which is the transport time of the aspirated gas through the tubing to the sample chamber, is generally not a problem because it can be measured and simply added to the response time shift (Figure 1). The only concern with the increasing lag time is gas mixing within the sampling tube, which blunts the response of the instrument. A similar lag and response time correction is applied to the measurement of the tracer gas concentration.

Helium was used as the tracer gas. Helium concentration was measured by a mass spectrometer. The advantages of the mass spectrometer are that very low aspiration rates are used (typically $1 \mathrm{~mL} / \mathrm{s}$ ), the response is rapid (typically $0 \%$ to $90 \%$ response of $80 \mathrm{~ms}$ ) and the signal to noise ratio is high (noise less than $0.1 \%$ full scale). The mass spectrometer cannot be used to measure carbon monoxide concentration because both carbon monoxide and nitrogen have a molecular mass of about 28. A stable isotope with a higher molecular mass, $\mathrm{C}^{18} \mathrm{O}$, has been used, but it is expensive and potentially noisy because the tiny signal for $\mathrm{C}^{18} \mathrm{O}$ falls directly between the very large peaks in the mass spectrum for nitrogen and oxygen. Dry test gas was used containing $0.3 \%$ carbon monoxide, $10 \%$ helium, $21 \%$ oxygen, balance nitrogen.

Methane $(0.3 \%)$ has also been used as a tracer gas (14) and measured by an infrared analyzer with similar problems as the measurement of carbon monoxide. Unfortunately, using methane in very low concentrations makes noise more of a problem. The increased solubility of methane can lead to an overestimation of lung volume, but the predicted effect is small (14).

The linearity of gas analyzers presents an additional prob- 
TABLE 2

\section{Error in DLCo ${ }^{\mathrm{SB}}$ due to carbon monoxide analyzer nonlinearity}

\begin{tabular}{|c|c|c|c|c|c|}
\hline & \multicolumn{5}{|c|}{ Nonlinearity (\%) } \\
\hline & 0 & 0.5 & 1.0 & 1.5 & 2.0 \\
\hline \%error (0 s bht) & 0 & 1.77 & 3.23 & 4.93 & 6.61 \\
\hline \multirow[t]{3}{*}{ \%error (10 s bht) } & 0 & 0.69 & 1.46 & 2.18 & 2.92 \\
\hline & \multicolumn{5}{|c|}{$\mathrm{DLco}^{\mathrm{SB}}$ (mL/min/torr) } \\
\hline & 10 & 20 & 30 & 40 & \\
\hline \%error (0 s bht) & 8.74 & 4.92 & 3.23 & 2.67 & \\
\hline \%error (10 s bht) & 3.74 & 2.04 & 1.46 & 1.17 & \\
\hline
\end{tabular}

A nonlinearity of the form $f(x)=a x^{2}+b x$ was simulated for the carbon monoxide data from a normal adult lung model with a single breath diffusing capacity of the lung for carbon monoxide (DLCOSB) of 30 $\mathrm{mL} / \mathrm{min} / \mathrm{torr}$. In the upper panel, the nonlinearity specifies the maximum deviation as a percentage of full scale. The error gives the percentage error in the measured $D\left\llcorner C O^{S B}\right.$ from simulated manoeuvres with $0 \mathrm{~s}$ and $10 \mathrm{~s}$ breath hold time (bht). The lower panel shows the interaction between the nonlinearity of the carbon monoxide analyzer and the $D\left\llcorner C O^{S B}\right.$ in the lung model. $D\left\llcorner C O^{S B}\right.$ was varied from 10 to $40 \mathrm{~mL} / \mathrm{min} / \mathrm{torr}$. The nonlinearity in the carbon monoxide analyzer for these simulations was $1 \%$ full scale. The percentage error in the measured $D\llcorner C O S B$ for $O \mathrm{~s}$ and $10 \mathrm{~s}$ breath hold manoeuvres is shown

lem. Typically, gas analyzers are checked at zero and at full scale. Table 2 shows the effects of nonlinearity of the carbon monoxide analyzer on DLco ${ }^{\mathrm{SB}}$ measurements. Note that the error in DLco ${ }^{\mathrm{SB}}$ due to analyzer nonlinearity is dependent upon the carbon monoxide concentration, and hence varies with both breath hold time and DLco ${ }^{\mathrm{SB}}$. The linearity of the analyzers should be checked regularly (see calibration section).

Gas flow: A pneumotach mounted in the wall of a bags-inbox system was used to measure flow $(8,9,11)$ and the signal was integrated to obtain volume. A \#3 Fleisch pneumotach with a $\pm 2 \mathrm{cmH}_{2} \mathrm{O}$ differential pressure transducer was used. Ambient room air always flows through the pneumotach. Linearizing tubes that are six times longer than the diameter of the pneumotach are used to maintain a uniform flow profile. Other systems use in-line pneumotachs or mass flow sensors that must be compensated for gases of different densities and temperatures. Nonlinearities may be compensated by digitally processing the flow signal rather than by using linearizing cones or screens. If slow flow rates are used for the single breath manoeuvre, recommended for the threeequation method, fewer problems are encountered in measuring flow. An alternative strategy is to use a spirometer to measure volume.

Breathing circuit: It is important to keep equipment dead space to a minimum because increasing the dead space of the breathing circuit will increase the response time of the system. The effectiveness of rapidly responding gas analyzers will be lost if the sampling ports are located in a part of the circuit distant from the mouth or where mixing is slow. When using continuous monitoring of gas concentration signals, the functional equipment dead space is the volume of the tubing between the gas sample ports and the mouth (Figure 2). The gas sample ports should, therefore, be located as close to the mouth as possible. A low dead space two-way valve, the

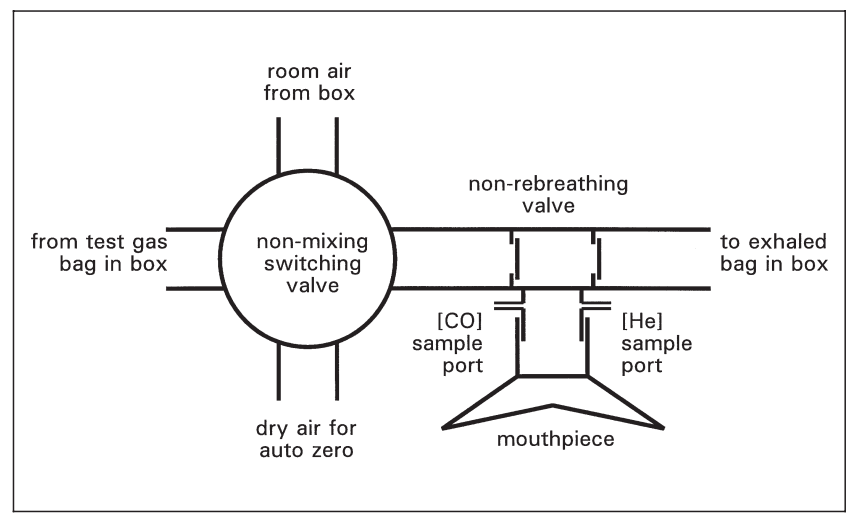

Figure 2) The breathing circuit for a bags-in-box system

inspiratory limb of which was connected to a three-way switching valve, was used with the gas sampling ports located just distal to the mouthpiece. Using an in-line flow sensor will increase the equipment dead space, but such devices are now available in small sizes with low resistance. These systems may use demand valves to deliver test gas, which require some negative pressure during inhalation, but it is unlikely that pressures will be in the range to influence diffusing capacity (15). The use of disposable filters will increase both dead space and response time.

It is very important to ensure that the system is free from leaks. With low gas concentrations and high aspiration rates, a small leak can make a significant difference in the signal. A method should be in place to check routinely for leaks. The method the authors use is to flush the system with medical air ( $21 \%$ oxygen, $79 \%$ nitrogen), close off the mouthpiece and blow test gas around the valves and tubing. If there is a leak then the meters will register an increase in carbon monoxide or helium concentration.

\section{CALIBRATION}

Flow meter: Because forced inhaled and exhaled flow rates are not used, the flow meter does not have to meet ATS requirements (3). The flow meter was checked for linearity by using a rotameter system. The flow meter was linear to within $1 \%$ of full scale over the range of 0.05 to $3 \mathrm{~L} / \mathrm{s}$ for both inhalation and exhalation. If a nonlinearity had been detected it would have been corrected with digital signal processing. On a daily basis, the flow signal is calibrated using a $3 \mathrm{~L}$ syringe. The first calibration check is done with the gas analyzers off so that they are not aspirating any gas. The calibration is accepted when the inspired and expired volumes measured from the syringe are within $1 \%$ of the syringe volume at flow rates of $0.1,0.5$ and $2 \mathrm{~L} / \mathrm{s}$.

The flow calibration is then repeated with the gas analyzers aspirating to compensate for the combined aspiration flow rate of the gas analyzers. A significant day to day change in the analyzer aspiration rate usually indicates a blockage, malfunction or leak in the system. The analyzer aspiration flow rate determined from the calibration is used to offset the flow signal during actual manoeuvres. The analyzer aspiration flow rate is also used to determine the 
point near the end of exhalation when the subject's exhaled flow rate falls below the analyzer aspiration rate. Since beyond this point the carbon monoxide and helium concentration data are meaningless, this becomes the end-of-test point for gas analysis.

Gas analyzers: For measurements of DLco ${ }^{\mathrm{SB}}$ the absolute accuracy of the analyzers is unimportant. Because ratios of gas concentrations are being compared, it is the relative accuracy, and hence the linearity of the meters, that is the main concern. The preferred method of determining the linearity of a gas analyzer is to deliver known dilutions of a given test gas to the analyzer and to compare the observed with the expected measurement. A Wöstoff Pump (Bochum, Germany) was used for this purpose. The linearities of the helium and the carbon monoxide concentration analyzers were measured separately. As part of the daily calibration routine, test gas is diluted with dry medical air and the output of the mass spectrometer is compared with the output of the carbon monoxide concentration analyzer (Figure 3). This procedure requires only a few minutes to perform because the output of the analyzers is sampled while the flow rates of the test gas and medical air are changed. This procedure would also be valid for dual gas analyzers (eg, infrared carbon monoxide and methane), which use different sensors for each gas.

Algorithm calibration: The authors have no device to calibrate the entire system. The hardware components are calibrated as described above. The software is verified by using data from a computer simulation of the single breath manoeuvre. Raw data from the analogue to digital converter are simulated and subsequently processed by the same software used to analyze real data. If the measured variables such as DLco $^{\mathrm{SB}}$ and lung volume are not the same as the simulated values for those variables over a range of expected operating conditions then the error in the software must be corrected.

\section{DATA ACQUISITION}

The analogue signals for flow, carbon monoxide concentration and helium concentration must be digitized for subsequent processing by the computer. The signals are filtered with a second-order $10 \mathrm{~Hz}$ low-pass filter and sampled at a rate of $50 \mathrm{~Hz}$ per channel. A 12 bit analogue to digital converter is used with the signal amplitude matched to the range of the converter. The effect of sampling rate was examined in lung models and healthy subjects. It was found that, for manoeuvres using flow rates less than $2 \mathrm{~L} / \mathrm{s}$, a rate of $50 \mathrm{~Hz}$ was adequate.

To guide the subject through the manoeuvre, a template of volume versus time was displayed for the prescribed manoeuvre, including the volume history control, on a computer screen. The subject's vital capacity (VC) and inspiratory capacity (IC) were measured in a practice manoeuvre without test gas. A real time tracing of the subject's volume versus time was displayed over the template. Most subjects and patients can easily adjust their flow rates to match the template with little practice. When the subject reached total lung capacity (TLC), the volume tracing was adjusted, if neces-

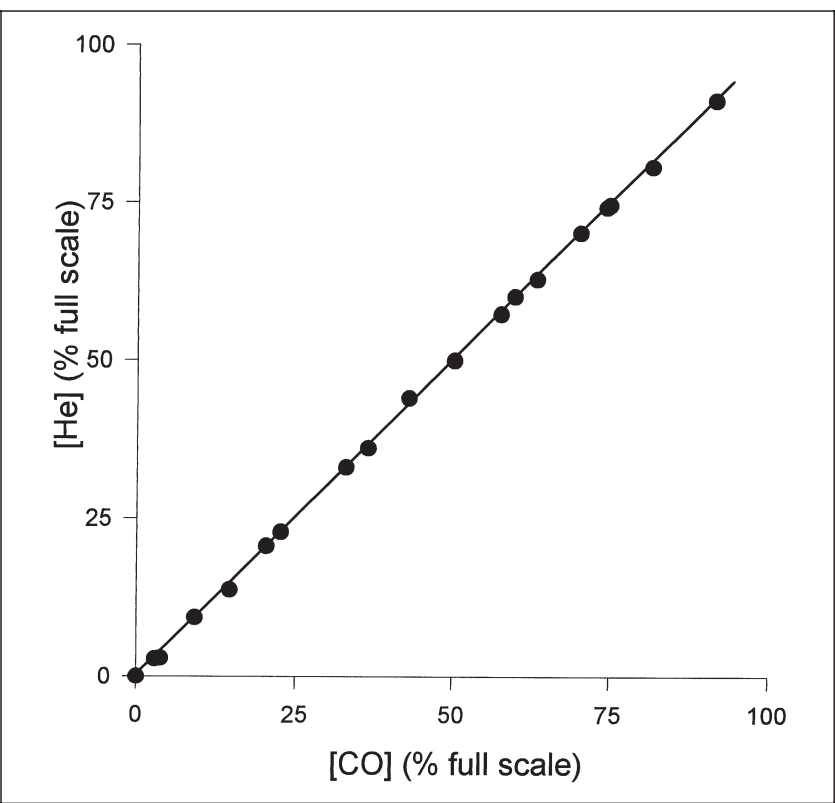

Figure 3) A typical check of analyzer nonlinearity comparing the carbon monoxide analyzer with the helium analyzer. Different dilutions of test gas are sampled simultaneously by the two analyzers. If either analyzer becomes nonlinear, the data points deviate from the line of identity

sary, to superimpose the template at TLC during the volume history control breath. The subject then exhaled to the premeasured functional residual capacity (FRC) to start inhalation of test gas using the template as a guide.

Washout between tests: When the subject first breathed through the mouthpiece, helium concentration was measured to ensure that adequate washout had occurred since the previous test. If the helium concentration rose above $0.1 \%$ within the first three tidal breaths then the test was aborted.

Zero levels: Before and after each single breath manoeuvre the average zero levels of the analyzers and flow meter over a $2 \mathrm{~s}$ period were determined with the flow meter tube occluded and the gas analyzers aspirating dry medical air. This gives a 'dry' zero for the carbon monoxide concentration analyzer with no carbon dioxide or water vapour. To control volume history each single breath manoeuvre was preceded by a standardized breath of room air with slow inhalation from FRC to TLC, a $5 \mathrm{~s}$ breath hold at TLC and slow exhalation back to FRC. During this deep breath, the ambient carbon monoxide concentration was measured during inspiration, and the premanoeuvre background carbon monoxide concentration in alveolar gas was measured during exhalation. The latter measurement was used as the 'wet' zero for the analyzer, incorporating any residual interference effects of water vapour and carbon dioxide in addition to background carbon monoxide, which includes carbon monoxide back pressure from smoking, environmental exposure or previous DLco ${ }^{\mathrm{SB}}$ tests. The 'wet' zero was applied to the carbon monoxide signal during the expiratory phase of the single breath manoeuvre, while the 'dry' zero was applied during the inspiratory phase. This strategy compensates for the back pressure of carbon monoxide, which is otherwise 


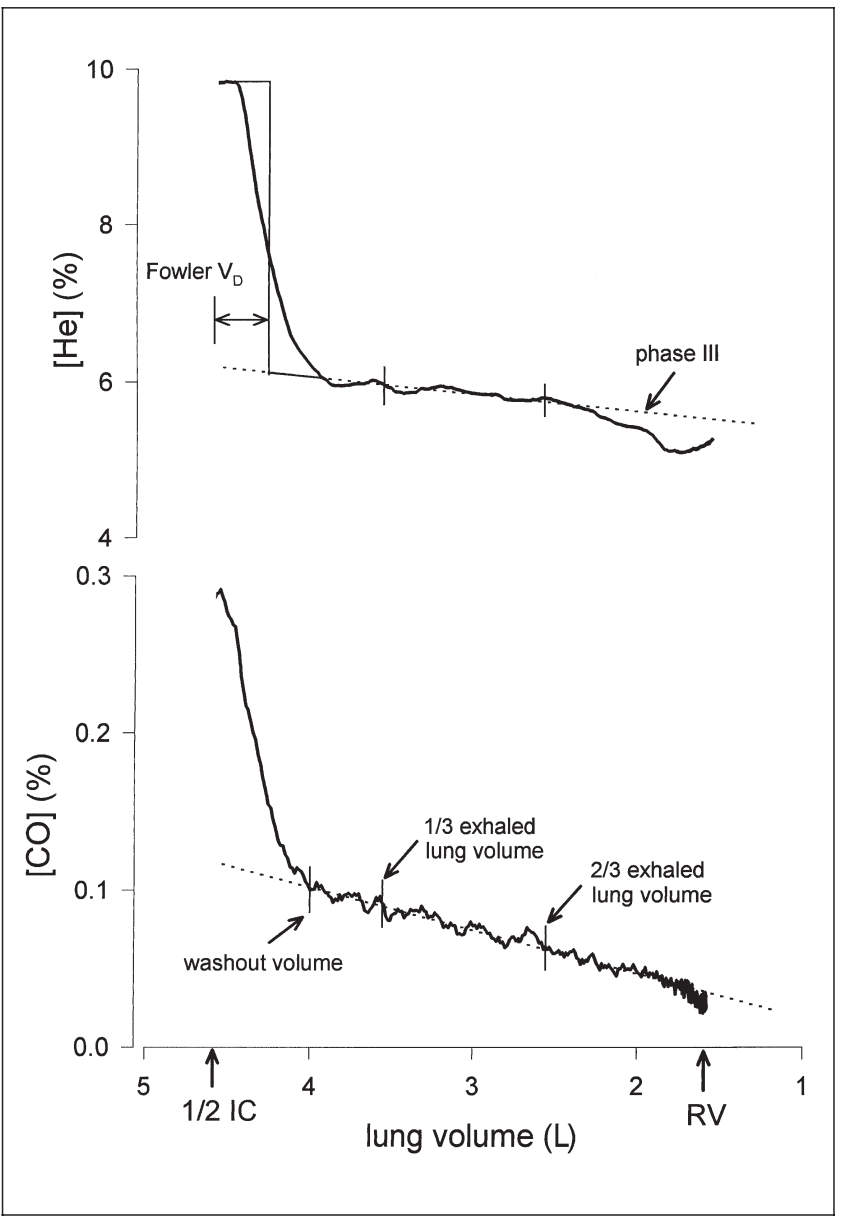

Figure 4) The Fowler dead space (VD) and the phase III slope are measured from the helium concentration washout curve (upper panel). The point of dead space washout is measured from the carbon monoxide concentration washout curve (lower panel). IC Inspiratory capacity; RV Residual volume

assumed to be zero and which can be a significant factor when analyzing serial single breath manoeuvres and especially when studying smokers.

The 'dry' zero baseline levels before and after the manoeuvre were compared. If the carbon monoxide concentration zero level drifted by more than 20 parts/million, the helium concentration zero level drifted by more than 300 parts/million or the flow zero level drifted by more than $10 \mathrm{~mL} / \mathrm{s}$, then the test was rejected.

Water vapour was removed from the gas sampled by the carbon monoxide analyzer using tubing that is selectively permeable to water vapour. The tubing is inside a sheath through which dry medical air is continuously flushed. While this eliminates most of the water vapour, the zero level procedure compensates for the small, residual interference.

Some commercial systems simply equilibrate water vapour to the ambient level in room air by aspirating the sample through water vapour permeable tubing exposed to room air. The above procedure of obtaining a 'wet' zero level should still be used to compensate for the back pressure of carbon monoxide and any residual interference of carbon dioxide and water vapour.

\section{LUNG VOLUMES AND DISTRIBUTION OF VENTILATION}

Volume: The measurement of DLco ${ }^{\mathrm{SB}}$ requires a measurement of absolute lung volume (VA). In conventional DLco ${ }^{\mathrm{SB}}$ methods, VA is estimated as inspired tracer gas concentration ([He]INSP) times inspired volume (VINSP) divided by tracer gas concentration in the exhaled gas sample ([He]SAMP). For example, for helium as tracer gas:

$$
\mathrm{VA}_{\mathrm{A}}=\frac{[\mathrm{He}] \mathrm{NSP} \cdot \mathrm{VINSP}}{[\mathrm{He}] \mathrm{SAMP}}
$$

The inherent assumption is that [He]SAMP is equal to the mean gas concentration in the lung at end-inspiration. Whereas this approximation may be reasonable in subjects with little ventilation inhomogeneity, it usually underestimates lung volume in the patients with lung disease (11). The lung volume measurement is also influenced by the timing of the exhaled gas sample because [He]SAMP will be smaller if the sample is collected later in exhalation.

If a continuous recording of the tracer gas is available, more information can be used to calculate VA. The algorithm the authors use (11) measures all of the tracer gas inhaled and all of the tracer gas exhaled to determine how much of the tracer gas remains in the lung at residual volume (RV). VA is calculated by assuming that the tracer gas concentration measured at end-expiration is equal to the mean concentration of tracer gas in the lung at RV.

$t_{0}$ was defined as the start of inhalation of test gas, $t_{1}$ as the end of inspiration - the point of maximum lung volume - and $t_{2}$ as the end of exhalation - the point when the expiratory flow falls to the level of the combined analyzer aspiration rate. $\mathrm{VA}$ at end-exhalation, $\mathrm{VA}\left(\mathrm{t}_{2}\right)$, is found as:

$$
V_{A}\left(t_{2}\right)=\int_{t_{0}}^{t_{2}} \frac{H e(t) d V(t)}{H e\left(t_{2}\right)}
$$

where $\mathrm{V}(\mathrm{t})$ is the lung volume at time $\mathrm{t}$ and $\mathrm{He}(\mathrm{t})$ is the fractional helium concentration at time t. To reduce noise, the mean of the last 10 sample points is used for $\mathrm{He}\left(\mathrm{t}_{2}\right)$. In contrast to conventional methods, this algorithm yields a robust calculation of VA that does not change with breath hold time (11). This calculation of lung volume includes the equipment dead space proximal to the gas sample ports, the anatomic dead space and the alveolar volume. The measured flow signal is converted to conditions of body temperature saturated with water vapour (BTPS) and, hence, VA is also BTPS.

Dead space: Anatomic dead space is determined by a computerized algorithm based on the Fowler method (16) with the obvious changes to accommodate the wash-in of a tracer gas rather than the washout of nitrogen. It was found that dead space changed with lung volume (10) in a similar manner to that previously reported (17). Dead space also varies with breath hold time $(10,11)$. This measurement of dead space includes the equipment dead space between the gas analyzer sample ports and the mouth. In the three-equation algorithm, dead space is subtracted from VA to give alveolar lung volume. 
Washout volume: The point of dead space washout was determined from the carbon monoxide concentration washout curve. A linear regression line through the middle third (by volume) of exhaled carbon monoxide versus lung volume was found. The point at which the observed carbon monoxde concentration first crossed this line was considered to be the point of dead space washout (Figure 4). The washout volume was the volume exhaled from maximum lung volume to this point. The washout volume varied with flow rate; exhaling at $0.5 \mathrm{~L} / \mathrm{s}$ resulted in a smaller washout volume than exhaling at $2 \mathrm{~L} / \mathrm{s}(10)$.

Phase III slope: The measurement of the slope of the alveolar plateau (phase III) of the tracer gas washout curve $(\Delta \mathrm{He} / \mathrm{L})$ was based on the single breath nitrogen washout method (18). A linear regression line through the middle third (by volume) of exhaled helium versus lung volume was found. The slope of this line was $\Delta \mathrm{He} / \mathrm{L}$. Because $\Delta \mathrm{He} / \mathrm{L}$ depends on the concentration of helium in the lung, which in turn depends on the specific ventilation (10), the slope is normalized to account for differences in specific ventilation. The predicted mean end-inspiratory alveolar helium concentration ([He]APRED), assuming perfect mixing, is calculated by the equation:

$$
[H e]_{\text {ApRed }}=\int_{t_{0}}^{t_{0}} \frac{H e(t) d V(t)}{V_{A}\left(t_{1}\right)}
$$

where $t_{D}$ is the time when $V(t)=V\left(t_{1}\right)-V D, V D$ is the Fowler dead space, and $\mathrm{VA}\left(\mathrm{t}_{1}\right)$ is the alveolar volume at end-inhalation. The normalized phase III slope $(\mathrm{Sn})$ is:

$$
\mathrm{Sn}=\frac{\Delta \mathrm{He} / \mathrm{L}}{[\mathrm{He}]_{\text {APRED }}}
$$

Sn was found to be independent of gas concentration, whereas $\Delta \mathrm{He} / \mathrm{L}$ increased with increasing helium concentration (10). The reproducibility of $\mathrm{Sn}$ was much better than that of $\Delta \mathrm{He} / \mathrm{L}$.

Mixing efficiency: The calculated mixing efficiency was similar to that of Cumming and Guyatt (19), but the authors used the wash-in of helium rather than washout of nitrogen (20). The mean exhaled helium concentration for all of the exhaled gas was found ([He]MEAS). The mixing efficiency (EMIX) was the ratio of the predicted to the observed mean helium concentration, ie:

$$
E_{M x}=\frac{[\text { He] }]_{\text {PREE }}}{[\text { He] }]_{\text {MAS }}}
$$

EMIX was found to be very reproducible, with a coefficient of variation of less than $0.5 \%(10,20,21)$.

\section{THREE-EQUATIONS ALGORITHM}

The equations for the uptake of carbon monoxide are all based on the general mass balance equation, which states that the change in the mass of carbon monoxide in the lung is equal to the mass of carbon monoxide diffusing out of the lung plus the mass of carbon monoxide coming into or out of the mouth, ie, change in carbon monoxide in lung $=-$ carbon monoxide diffused + carbon monoxide flow at mouth:

$$
\frac{d V(t) C O(t)}{d t}=-D L c o \cdot P A_{c o}(t)+C O_{m}(t) \frac{d V(t)}{d t}
$$

where $\mathrm{V}(\mathrm{t})$ is the absolute alveolar lung volume at time $\mathrm{t}$, $\mathrm{CO}(\mathrm{t})$ is the fractional concentration of carbon monoxide in the dry gas in the lung, $\mathrm{CO}_{\mathrm{m}}(\mathrm{t})$ is the fractional concentration of carbon monoxide in the dry gas at the mouth and $P \mathrm{Aco}_{\mathrm{co}}(\mathrm{t})$ is the partial pressure of carbon monoxide in the alveolar gas. If $\mathrm{P}_{\mathrm{B}}$ is the barometric pressure in torr then the partial pressure of the dry gas is $\mathrm{PD}_{\mathrm{D}}=\mathrm{PB}_{\mathrm{B}}-47$ and $\mathrm{Paco}_{\mathrm{c}}(\mathrm{t})=\mathrm{P}_{\mathrm{D}} \cdot \mathrm{CO}(\mathrm{t})$ For inhalation equation [1] becomes:

$$
V(t) \frac{d C O(t)}{d t}+C O(t) \frac{d V(t)}{d t}=-D L C O \cdot P_{D} \cdot C O(t)+C_{m}(t) \frac{d V(t)}{d t}
$$

During breath holding at some constant volume $\mathrm{V}_{\mathrm{BH}}$ equation 1 becomes

$$
V_{B H} \frac{d C O(t)}{d t}=-D L C O \cdot P_{D} \cdot C O(t)
$$

which is equivalent to the familiar Krogh equation (1). During exhalation, carbon monoxide concentration in the lung is assumed to be equal to the carbon monoxide concentration at the mouth. In the computer algorithm a time delay equal to the dead space transit time is used to eliminate this assumption. During exhalation, equation [1] becomes:

$$
V(t) \frac{d C O(t)}{d t}=-D L C O \cdot P_{D} \cdot C O(t)
$$

If inhalation occurs from time to to time $\mathrm{t}_{1}, \mathrm{CO}\left(\mathrm{t}_{0}\right)=0, \mathrm{f}(\mathrm{t})$ $=\mathrm{dV}(\mathrm{t}) / \mathrm{dt}$ and $\mathrm{CO}_{\mathrm{m}}(\mathrm{t})$ is monitored, then equation [2] can be solved to give the carbon monoxide concentration in the lung at end-inhalation (equation [5], below). If exhalation occurs from time $t_{1}$ to time $t_{2}$, then equation [2] can be solved to give the carbon monoxide concentration during exhalation, $\mathrm{CO}_{\mathrm{E}}(\mathrm{t})$ :

$$
\mathrm{CO}_{E}(\mathrm{t})=\mathrm{CO}\left(\mathrm{t}_{1}\right) \cdot \exp \left[-\mathrm{DLco} \cdot \mathrm{P}_{\mathrm{o}} \int_{\mathrm{t}_{1}}^{\mathrm{t}} \frac{\mathrm{dt}}{\mathrm{V}(\mathrm{t})}\right]
$$

where $t_{1} \leq t \leq t_{2}$. If a period of breath holding were to occur

$$
C O\left(t_{1}\right)=\exp \left[-\int_{t_{0}}^{t_{1}} \frac{f(t)+D L c o P_{D}}{V(t)} d t\right] \cdot \int_{t_{0}}^{t_{1}} \frac{C O_{m}(t) \cdot f(t)}{V(t)} \cdot \exp \left[\int_{t_{0}}^{t} \frac{f(t)+D L c o \cdot P_{D}}{V(t)} d t\right] d t
$$


from time $t_{x}$ to time $t_{y}$, then the carbon monoxide at the end of breath holding would be:

$$
\operatorname{CO}_{E}\left(t_{y}\right)=\operatorname{CO}_{E}\left(t_{x}\right) \cdot \exp \left(-\frac{D L c o \cdot P_{D} \cdot\left(t_{y}-t_{x}\right)}{V\left(t_{x}\right)}\right)
$$

However, during breath holding, both the equations 2 and 4 reduce to equation 3 . Therefore, in the implementation, only two equations, 5 and 6 , are used but the name 'threeequation method' has been retained to avoid confusion with previous reports. Any breath hold period occurring before or after the point of maximum volume will be adequately handled by equations 5 and 6 , respectively. This eliminates the need to define the start and end of breath holding for the calculation of DLco ${ }^{\mathrm{SB}}$.

Equation 6 can be used to predict the mean carbon monoxide concentration in the exhaled gas over a selected period $\mathrm{t}_{\mathrm{a}}$ to $\mathrm{t}_{\mathrm{b}}$, where $\mathrm{t}_{1}<\mathrm{t}_{\mathrm{a}}<\mathrm{t}_{\mathrm{b}}<\mathrm{t}_{2}$ :

$$
[C O]_{\text {PRED }}=\frac{\int_{t_{a}}^{t_{b}} \operatorname{CO}_{E}(t) d V(t)}{V\left(t_{b}\right)-V\left(t_{a}\right)}
$$

The mean carbon monoxide concentration over this same interval can be measured ([CO]MEAS). An iteration technique can now be used to solve the equations for DLco. A simple bisection method was chosen for stability. Although this method is slower than other iteration techniques, current computer processor speeds make the extra time for more iterations negligible.

The bisection method finds an upper and lower bound for DLco and uses the midpoint for succesive iterations. One starts by arbitrarily assuming DLco $=30 \mathrm{~mL} / \mathrm{min} /$ torr, STPD. If the initial value for DLco is too high then [CO]PRED will be lower than [CO]MEAS. Conversely, if the initial value for DLco is too low then [CO]PRED will be higher than

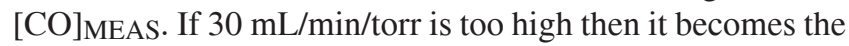
upper bound and 0 becomes the lower bound. If 30 $\mathrm{mL} / \mathrm{min} /$ torr is too low, then it becomes the lower bound. The DLco is then doubled until an upper bound is established.

Once the upper and lower bounds are found the new value for DLco is:

$$
\mathrm{DLCO}_{\text {new }}=\frac{\text { DLCOupper }+\mathrm{DLCO}_{\text {lower }}}{2}
$$

DLconew is then used to find [CO]PRED, which is compared

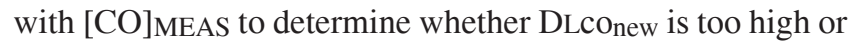
too low. If it is too high, then it becomes the upper bound for the next iteration. If it is too low then it becomes the lower bound.

This process is repeated until [CO]PRED is within a specified tolerance of $[\mathrm{CO}]$ MEAS. The authors use $0.1 \%$. The DLco value thus found is then converted to $\mathrm{mL} / \mathrm{min} /$ torr STPD. In most instances, 12 or fewer iterations are required to converge to the solution for DLco.

DLco is not permitted to be negative. If DLco $=0$ returns a value of [CO]PRED that is too low, then a value of zero is returned for DLco.

The algorithm was written in QUICKBASIC (Microsoft
Corporation, Washington). For a typical run, the lung volumes and diffusing capacity were calculated within $5 \mathrm{~s}$ using a PC type computer with a 486 central processor.

Sample size: A physical sample of alveolar gas is not collected in a bag for analysis. Rather, any specific sample can be constructed from the flow and gas concentration data. The largest exhaled gas sample for analysis begins at the point of dead space washout and continues to end-exhalation, which is defined as the point when the exhaled flow rate decreases to the level of the aspiration rate of the analyzers. The main advantages of using the largest possible exhaled gas sample are, first, the estimate of DLco ${ }^{\mathrm{SB}}$ will be more representative of the entire lung and less influenced by ventilation and/or diffusion inhomogeneities in the lung; and second, the measurement of VA will similarly be more accurate. Additionally, the effect of carbon monoxide analyzer noise will be diminished as the signal to noise ratio improves proportionally to the square root of the number of samples.

Index of diffusion inhomogeneity: The effects of diffusion inhomogeneity on measurements of DLco ${ }^{\mathrm{SB}}$ were recently described (21). The overall diffusing capacity was found by using the maximum size sample as described above. The large sample of alveolar gas was then divided into four equal volume samples and the data were reanalyzed four times, with a separate DLco ${ }^{\mathrm{SB}}$ calculated for each of the four smaller samples. The VA found from the maximum size sample was used for the subsequent analysis of the smaller samples. For submaximal manoeuvres with no breath holding, it was found that DLco ${ }^{\mathrm{SB}}$ measured from the samples collected early in exhalation were reduced while DLco ${ }^{\mathrm{SB}}$ from later samples was increased compared with the DLco ${ }^{\mathrm{SB}}$ from the total sample (21). With the addition of $6 \mathrm{~s}$ of breath holding the DLco $^{\mathrm{SB}}$ from the small exhaled alveolar gas samples were all the same as the DLco ${ }^{\mathrm{SB}}$ from the entire exhaled alveolar gas sample. There was no difference in the DLco ${ }^{\mathrm{SB}}$ from the entire exhaled sample for the 0 and 6 s breath hold manoeuvres.

The authors have since developed an index to quantify diffusion nonuniformity, (DI). The DLco ${ }^{\mathrm{SB}}$ for each small sample $\left(\mathrm{DLco}^{\mathrm{SB}_{\mathrm{i}}}\right.$ ) is normalized by expressing it as a percentage of the DLco ${ }^{\mathrm{SB}}$ for the large sample. DI is calculated as:

$$
D_{1}=\left[\frac{1}{4} \sum_{i=1}^{4}\left(\operatorname{DLCO}_{i} \mathrm{SB}_{i}-100\right)^{2}\right]^{\frac{1}{2}}
$$

Manoeuvre parameters: While this method can be applied to any single breath manoeuvre, the focus here is on a slow, submaximal manoeuvre. The single breath manoeuvre is done seated, immediately preceded by deep breath to control volume history. The control breath consists of inhaling room air from FRC to TLC, breath holding at TLC for $5 \mathrm{~s}$ and exhaling back to FRC. The single breath manoeuvre is then an inhalation of test gas from FRC to one-half of the IC and exhalation without breath holding to RV (Figure 5). All flow rates should be at a rate corresponding to $10 \%$ of the $\mathrm{VC} / \mathrm{s}$. Position: DLco ${ }^{\mathrm{SB}}$ measured in supine $(22)$ or $15^{\circ}$ head down (23) position is increased compared with sitting. Addition- 
ally, gravitationally dependent ventilation inhomogeneity is decreased in the supine position (23). However, the DLco ${ }^{\mathrm{SB}}$ in the upright seated position may be more reflective of ambient gas exchange in ambulatory persons. If further investigations are warranted, supine tests can be done to determine the amount of recruitment of pulmonary vasculature or increase in blood volume.

Volume history: An inhalation to TLC before the inhalation of test gas significantly increases the subsequent measurement of DLco ${ }^{\mathrm{SB}}$ compared with DLco ${ }^{\mathrm{SB}}$ measurements made after a 10 min control period of tidal breathing $(20,24,25)$. To control volume history, the choices are to precede the inhalation of test gas by either a deep breath or a controlled period of tidal breathing. Using a deep breath as the control has many advantages. It is easier to perform, easier to control and easier to standardize than tidal breathing. Because the effect of a deep breath can last for 3 mins (25), a long tidal breathing control period would be required. Patients instructed to breath normally on a mouthpiece typically hyperventilate. A deep breath also provides a TLC reference for subsequent submaximal manoeuvres and permits carbon monoxide back pressure measurements.

Inspired volume: The traditional manoeuvre starts at RV with inspiration to TLC. Starting at FRC and inhaling onehalf IC is closer to normal breathing than going from RV to TLC. In normal subjects, the DLco ${ }^{\mathrm{SB}}$ measured from this manoeuvre is about $10 \%$ lower than that measured from an RV-TLC manoeuvre $(10,24)$. While a VC inhalation minimizes ventilation nonuniformities in the lung and would be easier to standardize in the clinical setting, a submaximal breath from FRC is more sensitive in the detection of peripheral inhomogeneities in the lung, which are usually worse in disease (10). A possible problem with inhaling a small volume of test gas is a greater dilution of test gas and consequently reduced signal to noise ratios. This is partially offset by the reduction in breath hold time. Further enhancement may be possible by using higher concentrations of carbon monoxide and tracer gas in the test gas.

Breath hold time: Eliminating breath holding makes the manoeuvre more natural and easier to perform. It also makes the measurement of DLco ${ }^{\mathrm{SB}}$ and lung mechanics sensitive to gas mixing inhomogeneities, which are not observed with increased breath hold times $(10,20,21)$. If warranted for a given patient with maldistribution of ventilation, a second measurement can be made at 6 to $10 \mathrm{~s}$ of breath holding to determine whether increased gas mixing alters DLco ${ }^{\mathrm{SB}}$. For purposes of describing the manoeuvre, the breath hold time is defined as the interval from $100 \mathrm{~mL}$ below TLC on the inspiratory limb to $100 \mathrm{~mL}$ below TLC on the expiratory limb.

Exhaled volume: One of the strengths of the three-equation method is the ability to include all of the exhaled gas data in the calculation of DLco ${ }^{\mathrm{SB}}$. The largest possible exhaled volume gives the best mean estimate of DLco ${ }^{\mathrm{SB}}$. For this reason exhalation should be done to RV. Exhaling to RV also provides measurements of $\mathrm{VC}$ and $\mathrm{RV}$ from the same manoeuvre.

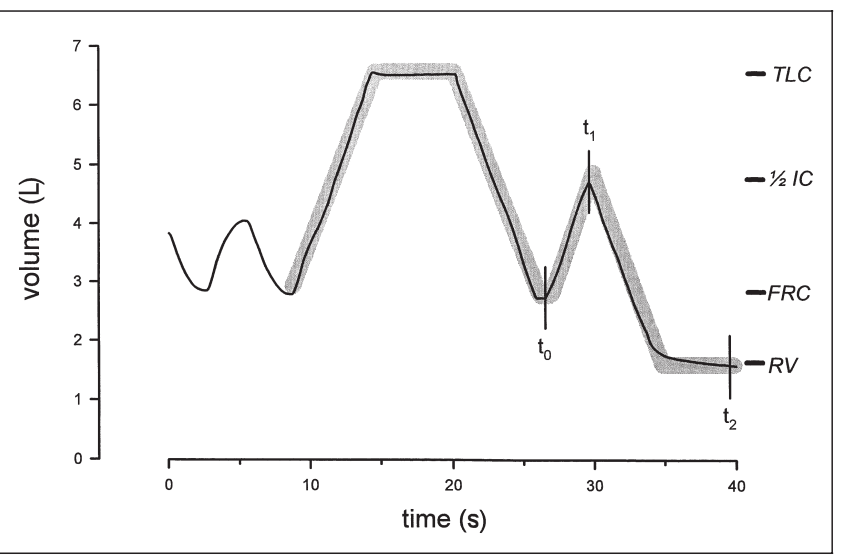

Figure 5) Functional residual capacity (FRC) is determined from tidal breathing before the manoeuvre. A standard deep breath of room air to total lung capacity (TLC) with breath holding for $5 \mathrm{~s}$ and exhalation back to the premeasured FRC is done for volume history control. The subject then inhales test gas from FRC to half inspiratory capacity $(1 / 2 I C)$ and exhales to residual volume $(R V)$. All flow rates are slow, $10 \%$ of the vital capacity per second. The subject matches a real-time display of volume-time to a template of the manoeuvre (shaded area) on the computer screen to attain the desired volumes and flow rates

Flow rate: A slow flow rate is not only more physiological but permits the single breath manoeuvre to be done equally well by normal subjects and patients with obstructive lung disease. Much of the work done by the authors has used a flow rate of $0.5 \mathrm{~L} / \mathrm{s}$, which was arbitrarily determined. For children and subjects with low volumes, a flow rate of $10 \%$ of the VC per second was used. Standardizing the flow rate to a fraction of the $\mathrm{VC}$ rather than a fixed flow rate will make the manoeuvre more comparable between subjects with different lung volumes.

A disadvantage of using manoeuvres with high flow rates is that far more rapidly responding gas analyzers are required because a significant volume may be exhaled during the transient response time of the analyzer. This may have been one of the reasons for the increased washout volumes reported by Huang and MacIntyre (14), who studied standardized (rapid) single breath manoeuvres with a $300 \mathrm{~ms}$ response time analyzer.

'Intrabreath' measurements of DLco: The three-equation method is not to be confused with measurements of DLco during exhalation, referred to as 'DLco(exhaled)', 'intrabreath DLco' or 'within-breath DLco'. Newth et al (26) described a technique of measuring DLco during exhalation based on an approximation of the Krogh equation. The authors showed that this method was influenced by ventilation inhomogeneity, causing the measured DLco to vary with exhaled flow rate (27). Additionally, such measurements are very sensitive to noise in the monitored carbon monoxide signal. The authors proposed using a discrete sample method of measuring DLco(exhaled) (28), one variation of which used all of the exhaled data to determine a single value of DLco(exhaled). Huang et al (29) reported measurements of DLco(exhaled) using the analysis of Martonen and Wilson (30). This method also has the advantage of using all of the 
exhaled gas to calculate a single value of DLco(exhaled), but relies on the assumption of a linear decline in $\ln [\mathrm{CO}]$ with lung volume and a constant exhaled flow rate. It was found that intrabreath methods of measuring DLco, which essentially measure the slope of the exhaled carbon monoxide concentration curve, are spuriously affected by ventilation inhomogeneity, cannot reliably measure DLco and cannot distinguish between nonuniform ventilation and nonuniform diffusion in the lung (31).

\section{DISCUSSION}

While the measurement of DLco ${ }^{\mathrm{SB}}$ has been used extensively for clinical and research purposes, the full potential of its value has never been realized. The limitations of conventional DLco ${ }^{\mathrm{SB}}$ measurements have led to spurious variations in the results due to variations in flow rates, breath hold times, breath hold volume, and the size and timing of the alveolar gas sample. Using separate, analytic equations to describe the total manoeuvre eliminates these errors and removes the constraint of performing an unnatural, standardized manoeuvre that is very difficult for some patients to perform adequately.

The concept in our method that has been challenged most frequently is the assumption that, within a given single breath manoeuvre, DLco ${ }^{\mathrm{SB}}$ is constant and does not vary with time or lung volume. Conventional methods are based on the assumption that all the carbon monoxide uptake occurs at peak lung volume with a constant DLco ${ }^{\mathrm{SB}}$. While we could have assumed DLco ${ }^{\mathrm{SB}}$ to be some function of volume or time, results from normal subjects suggest that this is not the case $(8,9,11)$. Assuming DLco ${ }^{\mathrm{SB}}$ to be a constant yields the most useful results.

The effect of this assumption is that the DLco ${ }^{\mathrm{SB}}$ that is calculated by our method gives the mean diffusing capacity over the entire manoeuvre that would explain the observed uptake of carbon monoxide in a uniform, homogeneous lung. Each manoeuvre is analyzed separately so that DLco ${ }^{\mathrm{SB}}$ is measured independently in each single breath manoeuvre. Hence, the assumption that DLco ${ }^{\mathrm{SB}}$ does not vary with lung volume within a given single breath manoeuvre does not prevent the study of how DLco ${ }^{\mathrm{SB}}$ changes with lung volume from manoeuvre to manoeuvre. If there were a decrease in DLco $^{\mathrm{SB}}$ with lung volume, then a lower rate of carbon monoxide uptake would be observed for a manoeuvre done at a lower lung volume, and a lower DLco ${ }^{\mathrm{SB}}$ would be calculated.

In normal subjects, DLco ${ }^{\mathrm{SB}}$ increases slightly as peak lung volume increases, but is not affected by changes in the timeweighted mean lung volume. At short breath hold times mean lung volume can be much less than peak lung volume, while if inhalation and exhalation are done very rapidly and the breath hold is long then mean lung volume approaches peak lung volume. We showed previously that DLco ${ }^{\mathrm{SB}}$ is not affected by breath hold time in normal subjects, and is thus independent of mean lung volume (11). The effect of peak lung volume on DLco ${ }^{\mathrm{SB}}$ is nonlinear and is dependent on volume history (24). DLco ${ }^{\mathrm{SB}} / \mathrm{VA}$ varies with lung volume (24).
In equations 1 to 4 we could have assumed that DLco ${ }^{\text {SB }}$ was some function of time and/or lung volume. Saidel et al (32) used the assumption that diffusing capacity was directly proportional to surface area, which, assuming a spherical compartment, would be proportional to volume to the twothirds power. In our lung models, we obtained the best fit of simulated to observed data from normal subjects when DLco was constant (7). In patients with lung disease, this may not be the case. Saidel and Modarreszadeh (33) found that DLco $^{\mathrm{SB}}$ was relatively independent of lung volume in normal subjects, but it became more volume-dependent in patients with chronic obstructive pulmonary disease. We found that the observed pattern of carbon monoxide uptake and tracer gas washout in patients with emphysema could be modelled with constant DLco ${ }^{\mathrm{SB}}$ but with the introduction of incomplete mixing of gas in the lungs.

We have shown that DLco ${ }^{\mathrm{SB}}$ measured by the Jones and Meade method (5) in normal subjects who can perform the standardized manoeuvre very well is very similar to the three-equation DLco ${ }^{\text {SB }}$ (9). Beck at al (12) also found that, in patients who adequately performed the standardized singlebreath manoeuvre, the three-equation analysis applied to an ATS-type sample of exhaled gas yielded similar results to a Jones and Meade analysis of the same sample. This implies that, until such time as a normal population-based study can be done to derive normal regression formulas, current normal regressions developed using conventional methods may be applied as a reasonable approximation. As recommended by the ATS, each laboratory should do a study of normal persons to verify the applicability of any set of regression equations (3).

An important improvement to the measurement of DLCo $\mathrm{SB}$ that is afforded by the technology of continuous monitoring of exhaled concentrations is the determination of the point of dead space washout. This eliminates the necessity of using an arbitrary volume to be discarded before the accumulation of an alveolar sample. This alone improves the precision of conventional methods (14) because there can be considerable variation in the gas required to wash out dead space (9). Continuous monitoring also allows the calculation of the Fowler dead space, which can be subtracted from the measured total lung volume to estimate alveolar lung volume. The Fowler dead space is not constant for any individual but varies with lung volume, flow rate and breath hold time $(17,20)$. The Fowler dead space provides an estimate of the portion of the measured lung volume that is not participating in gas exchange. This is a measure of functional rather than 'anatomic' dead space.

While breath hold time has little effect on DLco ${ }^{\mathrm{SB}}$ in normal subjects, DLco ${ }^{\mathrm{SB}}$ increases with increasing breath hold time in patients with emphysema and in patients with asthma (11). The index of diffusion uniformity, DI, changes much more with breath hold time in patients and in smokers than in normal subjects. Smokers with normal DLco ${ }^{\mathrm{SB}}$ have abnormal DI. In the assessment of some patients it may be useful to measure DLco ${ }^{\mathrm{SB}}$ at both $0 \mathrm{~s}$ and $6 \mathrm{~s}$ of breath hold to determine the degree of diffusion inhomogeneity. 
In summary, measurements of DLco ${ }^{\mathrm{SB}}$ using the threeequation method can potentially improve both the precision and the accuracy of DLco ${ }^{\mathrm{SB}}$ while permitting the measurement to be made from a single breath manoeuvre, which can be done equally well by normal subjects and by patients with lung disease, and which far more closely resembles normal respiration. These improvements may help to achieve more of the potential value for DLco ${ }^{\mathrm{SB}}$ as an important index of lung function.

ACKNOWLEDGEMENTS: The authors gratefully acknowledge research grant support from the Saskatchewan Lung Association and the Medical Research Council of Canada.

\section{REFERENCES}

1. Krogh M. The diffusion of gases through the lungs of man. J Physiol (Lond) 1914;49:271-300.

2. Cotes JE, Chinn DJ, Quanjer PH, Roca J, Yernault JC. Standardization of the measurement of transfer factor (diffusing capacity). Eur Respir J $1993 ; 6: 1-52$

3. American Thoracic Society. Single breath carbon monoxide diffusing capacity (transfer factor). Am J Respir Crit Care Med 1995;152:2119-26.

4. Ogilvie CM, Forster RE, Blakemore WS, Morton JW. A standardized breathholding technique for the clinical measurement of diffusing capacity by the single breath method. J Clin Invest 1957;36:1-17.

5. Jones RS, Meade F. A theoretical and experimental analysis of anomalies in the estimation of pulmonary diffusing capacity by the single breath method. Q J Exp Physiol 1961;46:131-43.

6. Ferris BG. Epidemiology standardization project. Am Rev Respir Dis 1978;118S:62-72.

7. Graham BL, Dosman JA, Cotton DJ. A theoretical analysis of the single breath diffusing capacity for carbon monoxide. IEEE Trans Biomed Eng 1980;BME-27:221-7.

8. Graham BL, Mink JT, Cotton DJ. Overestimation of single breath carbon monoxide diffusing capacity in patients with airflow obstruction. Am Rev Respir Dis 1984;129:403-8.

9. Graham BL, Mink JT, Cotton DJ. Improved accuracy and precision of single-breath CO diffusing capacity measurements. J Appl Physiol 1981;51:1306-13.

10. Cotton DJ, Prabhu MB, Mink JT, Graham BL. Effects of ventilation inhomogeneity on DLco ${ }^{\mathrm{SB}}$-3EQ in normal subjects. J Appl Physiol 1992;73:2623-30.

11. Graham BL, Mink JT, Cotton DJ. Effect of breath hold time on DLco ${ }^{\text {SB }}$ in patients with airway obstruction. J Appl Physiol 1985;58:1319-25.

12. Beck KC, Offord PO, Scanlon PD. Comparison of four methods of calculating diffusing capacity by the single breath method. Chest 1994;105:594-600.

13. Graham BL, Buchanan PR, Withy SJ, Harris EA. Data acquisition from a multiplex quadrupole mass spectrometer. Clin Phys Physiol Meas 1985;6:17-25.

14. Huang Y-CT, MacIntyre NR. Real-time analysis improves the measurement of single breath diffusing capacity. Am Rev Respir Dis 1992;146:946-50.

15. Cotton DJ, Mink JT, Graham BL. Effect of high negative inspiratory pressure on single breath CO diffusing capacity. Respir Physiol 1983;54:19-29.

16. Fowler WS. Lung function studies II. The respiratory dead space. Am J Physiol 1948;154:405-16.

17. Anthonisen NR, Fleetham JA. Ventilation: total, alveolar, and dead space. In: Fishman AP, ed. Handbook of Physiology Section 3: The Respiratory System, Volume IV - Gas Exchange. Baltimore: Waverly Press, 1987:13-29.

18. Craven N, Sidwall S, West P, McCarthy DS, Cherniack RM. Computer analysis of the single breath nitrogen washout curve. Am Rev Respir Dis 1976;113:445-9.

19. Cumming G, Guyatt AR. Alveolar gas mixing efficiency in the human lung. Clin Sci 1982;62:541-7.

20. Prabhu MB, Mink JT, Graham BL, Cotton DJ. Effect of a deep breath on gas mixing and diffusion in the lung. Respir Physiol 1990;79:195-204.

21. Soparkar GR, Mink JT, Graham BL, Cotton DJ. Measurement of temporal changes in DLco ${ }^{\mathrm{SB}}$-3EQ from small alveolar samples in normal subjects. J Appl Physiol 1994;76:1494-501.

22. Stam H, Kreuzer FJA, Versprille A. Effect of lung volume and positional changes on pulmonary diffusing capacity and its components. J Appl Physiol 1991;71:1477-88.

23. Cotton DJ, Graham BL, Mink JT. Pulmonary diffusing capacity in adult cystic fibrosis: reduced positional changes are partially reversed by hyperoxia. Clin Invest Med 1990;13:82-91.

24. Cotton DJ, Taher F, Mink JT, Graham BL. Effect of volume history on changes in DLco ${ }^{\mathrm{SB}}$-3EQ with lung volume in normal subjects. J Appl Physiol 1992;73:434-9.

25. Lebecque P, Mwepu C, Veriter C, Rodenstein D, Nemery B, Frans A. Hysteresis of the alveolar capillary membrane in normal subjects. J Appl Physiol 1986;60:1442-5.

26. Newth CJL, Cotton DJ, Nadel JA. Pulmonary diffusing capacity measured at multiple intervals during a single exhalation in man. J Appl Physiol 1977;43:617-25.

27. Cotton DJ, Graham BL. Effect of ventilation and diffusion nonuniformity on DLco(exhaled) in a lung model. J Appl Physiol 1980;48:648-56.

28. Graham BL, Mink JT, Cotton DJ. Dynamic measurements of CO diffusing capacity using discrete samples of alveolar gas. J Appl Physiol 1983;54:73-9.

29. Huang Y-CT, Helms MJ, MacIntyre NR. Normal values for single exhalation diffusing capacity and pulmonary capillary blood flow in sitting, supine positions, and during mild exercise. Chest 1994;105:501-8.

30. Martonen TB, Wilson AF. Theoretical basis of single breath gas absorption tests. J Math Biol 1982;14:203-20.

31. Cotton DJ, Prabhu MB, Mink JT, Graham BL. Effect of ventilation inhomogeneity on 'intra-breath' diffusing capacity [DLco(exhaled)] in normal subjects. J Appl Physiol 1993;75:927-32.

32. Saidel GM, Militano TC, Chester EH. A theoretical basis for assessing pulmonary membrane transport. Bull Physiopathol Respir 1973;9:481-96.

33. Saidel GM, Modarreszadeh M. Pulmonary diffusing capacity in the presence of ventilation inhomogeneity. J Appl Physiol 1987;63:2438-49. 


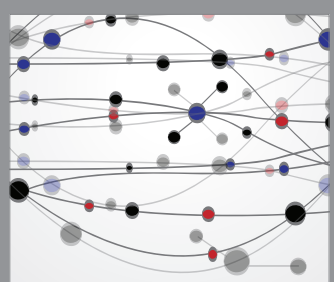

The Scientific World Journal
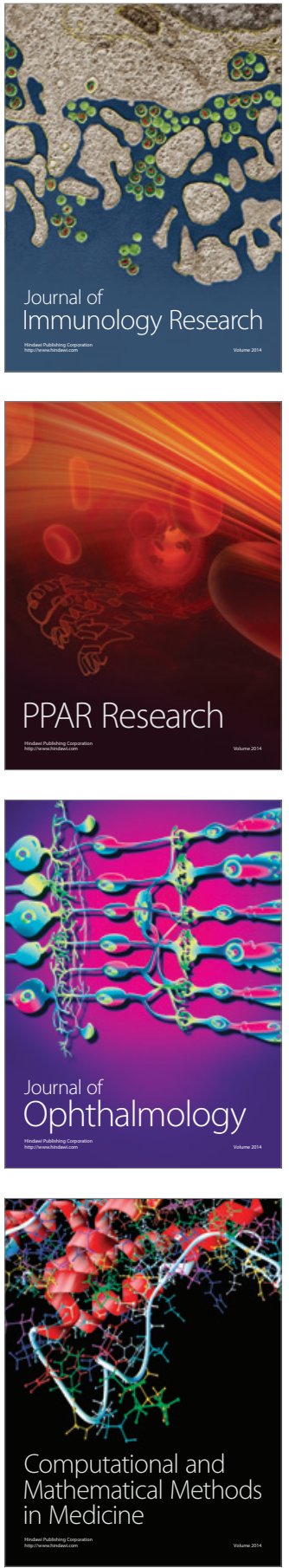

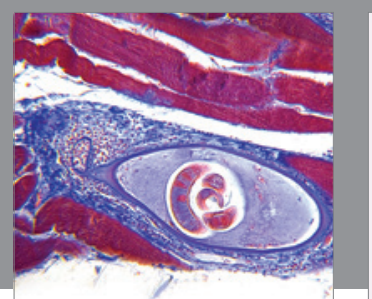

Gastroenterology Research and Practice

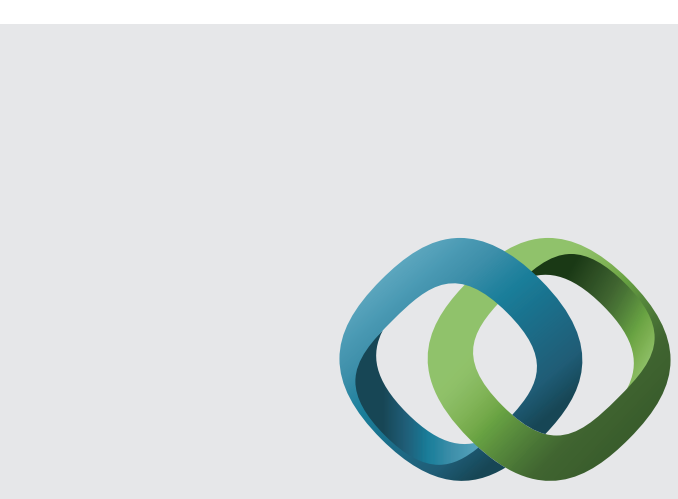

\section{Hindawi}

Submit your manuscripts at

http://www.hindawi.com
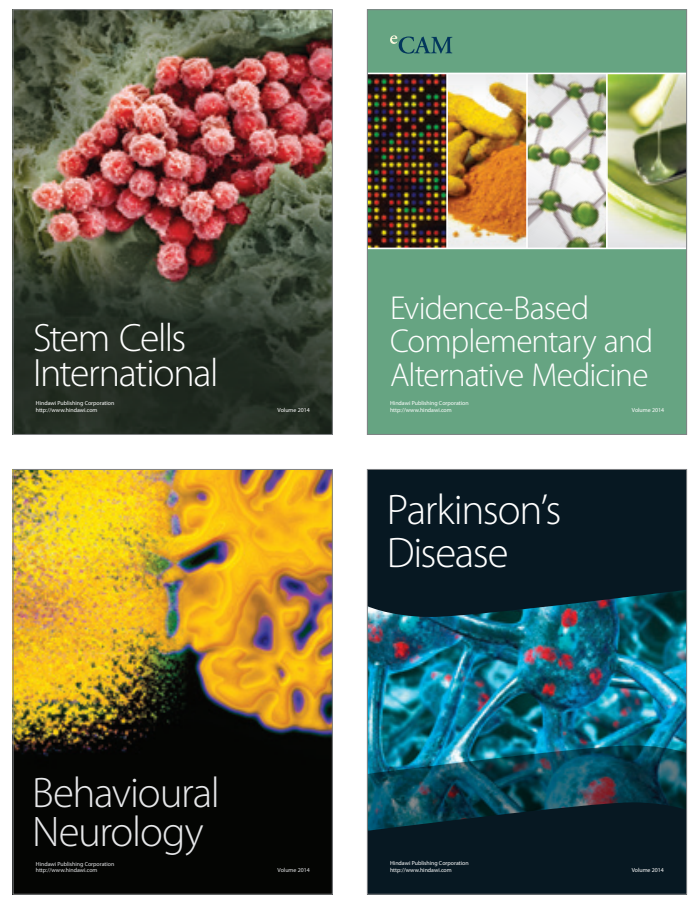
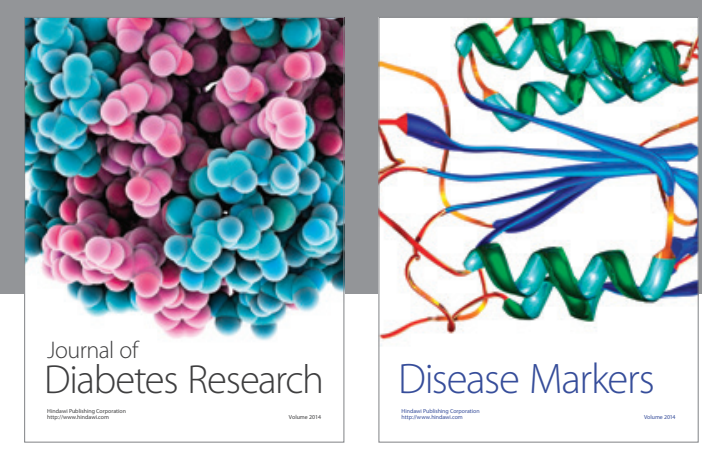

Disease Markers
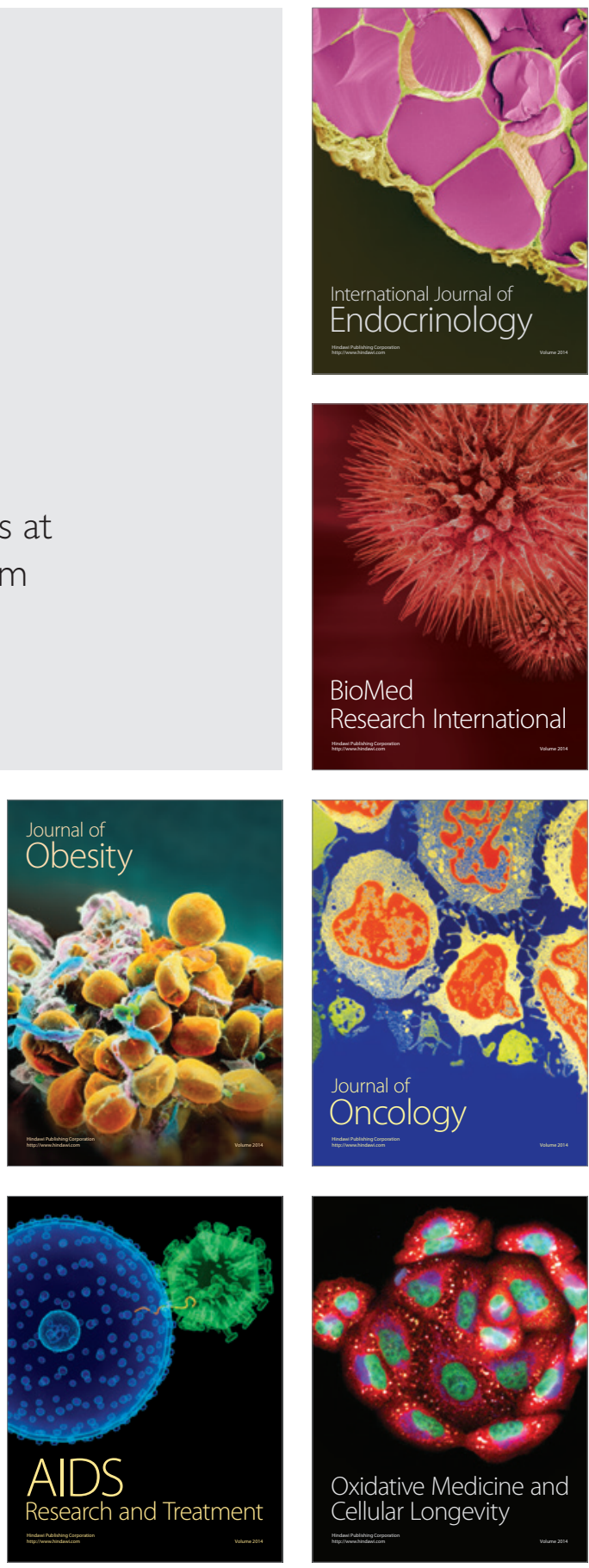